\title{
Bei welchen Indikationen reduziert die Tomosynthese die Recallrate?
}

Die dreidimensionale Tomosynthese der Brust erhöhte die Detektionsrate, verbesserte die Lokalisationsdiagnostik und reduzierte die Recallrate bei Patienten mit einem pathologischen Mammografiebefund. Bislang war unklar, ob dies für alle Auffälligkeiten gleichermaßen gilt. Radiology 2015; 274: 85-91

In der Studie von Melissa Durand und Kollegen von der Yale University School of Medicine werteten 7 Radiologen retrospektiv 17955 digitale Screening-Mam- mografien aus. Davon waren 9364 (52,2\%) 2-dimensionale Mammografien und 8591 $(47,8 \%)$ kombinierte 2- und 3-dimensionale Untersuchungen. 1154 und $671 \mathrm{~Pa}-$ 
tientinnen hatten auffällige Befunde, die eine weitere Abklärung erforderten. Dies entsprach einer Recallrate von $12,3 \%$ und $7,8 \%$ bzw. einer Reduktion in der Tomosynthese-Gruppe um 36,6\% ( $p<0,0001)$. Unter Berücksichtigung des Lebensalters, der Brustdichte und Tumorrisikofaktoren war das Risiko für einen Recall in der Standardgruppe 1,76-fach höher. Auf die Untersucher bezogen hatten 5 der 7 Spezialisten mit dem kombinierten Vorgehen geringere Rückrufraten. Die beiden anderen Ärzte waren diejenigen mit der kürzesten und mit der längsten Mammografie-Erfahrung.

Verglichen mit dem Standard wurden Patienten, die zusätzlich die Tomosynthese erhielten, signifikant seltener wegen Asymmetrien benachrichtigt $(3,1 \%$ vs. 7,4\%; Odds-Ratio OR 2,7; 95\%-Konfidenzintervall $\mathrm{KI} 2,3-3,1 ; \mathrm{p}<0,0001)$. Dies sei auf den Nachweis von Überlagerungsphänomen mit der Tomosynthese zurückzuführen. Auch Patientinnen mit Kalzifikationen profitierten, die Recallraten betrugen $2,4 \%(2 D+3 D)$ und 3,2\% (2D). Die Wahrscheinlichkeit für einen Rückruf wegen einer unklaren Verkalkungsstruktur war nach der konventionellen Mammografie 1,4-fach häufiger als nach Mammografie und Tomosynthese. Bei Tumormassen und anderen Veränderungen der Gewebearchitektur waren die Recallraten vergleichbar.

In der Tomosynthesegruppe wurden 51 Karzinome $(0,59 \%)$ und in der Standardgruppe 54 Karzinome (0,57\%) diagnostiziert. Der Unterschied war nicht signifikant $(\mathrm{p}=0,88)$. Auch im Vergleich mit historischen Kontrollen war die Detektionsrate mit der zusätzlichen Tomosynthese am höchsten $(0,44 \%)$, aber nicht signifi- kant verschieden ( $\mathrm{p}=0,12)$. Für die histologischen Subtypen bestanden Abweichungen. Mit dem kombinierten Vorgehen stellten sich mehr Architekturanomalien als invasive Karzinomen heraus. In beiden Gruppen waren diese meist $<2 \mathrm{~cm}$ und nodal negativ.

\section{Fazit}

Nach der Tomosynthese waren Rückrufe wegen auffälliger Mammabefunde seltener, so die Autoren. Dies galt insbesondere für Asymmetrien und Kalzifikationen.

Dr. med. Susanne Krome, Melle 